\title{
EVALUACIÓN DE UN CEBO FEROMONAL PARA LA CAPTURA DEL PICUDO DEL AGAVE (COLEOPTERA: CURCULIONIDAE)
}

\author{
HILDA RODRÍGUEZ-REBOLLAR, ${ }^{1}$ JULIO C. ROJAS, ${ }^{2}$ HÉCTOR GONZÁLEZ- \\ HERNÁNDEZ, ${ }^{1 *}$ LAURa DELIA ORTEGA-ARENAS, ${ }^{1}$ ARMANDo \\ EQUIHUA-MARTÍNEZ, ${ }^{1}$ JOSÉ IGNACIO DEL REAL-LABORDE ${ }^{3}$ \& JOSÉ \\ LÓPEZ-COLLADO ${ }^{4}$ \\ ${ }^{1}$ Programa de Entomología y Acarología, Colegio de Postgraduados, Carretera México-Texcoco Km \\ 36.5, Montecillo, Texcoco, Edo. de México, CP 56230. \\ ${ }^{2}$ Departamento de Entomología Tropical, El Colegio de la Frontera Sur (ECOSUR), Carretera Antigua \\ Aeropuerto Km. 2.5. Tapachula, Chiapas. \\ ${ }^{3}$ Rancho El Indio, Tequila Sauza. S. A. de R. L. de C. V., Tequila, Jalisco. \\ ${ }^{4}$ Colegio de Postgraduados, Campus Veracruz, km 88.5 carretera federal Xalapa-Veracruz, CP 91690, \\ Veracruz, Veracruz. \\ *Autor para correspondencia: <hgzzhdz@colpos.mx>
}

Rodríguez-Rebollar, H., J. C. Rojas, H. González-Hernández, L. D. Ortega-Arenas, A. EquihuaMartínez, J. I. del Real-Laborde \& J. López-Collado. 2012. Evaluación de un cebo feromonal para la captura del picudo del agave (Coleoptera: Curculionidae). Acta Zoológica Mexicana (n. s.), 28(1): 73-85.

RESUMEN. El picudo del agave, Scyphophorus acupunctatus Gyllenhal, es una plaga de importancia económica en diferentes cultivos de agaves en México, principalmente en agave tequilero, agave mezcalero y henequén. Los machos de esta especie liberan una feromona de agregación que atrae a ambos sexos, la feromona esta compuesta por dos alcoholes y dos cetonas. Experimentos previos han mostrado que los cuatro compuestos son atractivos a los picudos del agave, pero las mayores capturas se obtuvieron cuando se combinaron la 2-metil-4-heptanona (C1) y la 2-metil-4-octanona (C2). En el presente estudio se realizaron experimentos en campo para evaluar el efecto de las proporciones $(1: 1,1: 2,1: 4$, $2: 1$ y $4: 1)$ y la dosis $(50,100,150,200,350$, y $500 \mathrm{mg})$ de C1 y C2 en la captura de los picudos. Los resultados muestran que las diferentes proporciones probadas de los dos componentes feromonales no afectaron las capturas de los insectos. De hecho las trampas cebadas con C2 tuvieron mayores capturas que trampas cebadas con la combinación de los dos compuestos. Con respecto al otro factor evaluado, nuestros resultados muestran que la dosis no afectó las capturas de trampas cebadas con la feromona. Las trampas cebadas con los compuestos feromonales sintéticos capturaron más hembras que machos.

Palabras clave: Agave tequilana, Scyphophorus acupunctatus, cebo feromonal, dosis, monitoreo, trampeo masivo.

Recibido: 13/12/2010; aceptado: 25/10/2011. 
Rodríguez-Rebollar, H., J. C. Rojas, H. González-Hernández, L. D. Ortega-Arenas, A. EquihuaMartínez, J. I. del Real-Laborde \& J. López-Collado. 2012. Evaluation of a pheromone lure for capturing the agave weevil (Coleoptera: Curculionidae). Acta Zoológica Mexicana (n. s.), 28(1): 73-85.

ABSTRACT. The agave weevil, Scyphophorus acupunctatus Gyllenhal, is considered the most important insect pest of the cultivated agaves in Mexico. Recent studies on the chemical ecology of this weevil have shown that males release a pheromone that attracts both sexes; the pheromone is composed of four components. Previous field experiments showed that all components, singled or in blends, were attractive to the weevils, but compounds 2-methyl-4-heptanone $(\mathrm{C} 1)$ and 2-methyl-4-octanone $(\mathrm{C} 2)$ were sufficient to obtain captures equivalent to those by the quaternary blend. In this study, we investigated the effect of different ratios (1:1, 1:2, 1:4, 2:1, and 4:1) and doses $(50,100,150,200,350$, and $500 \mathrm{mg})$ of $\mathrm{C} 1$ and $\mathrm{C} 2$ in the captures of the agave weevils in commercial plantations of Agave tequilana Weber. Our results showed that traps baited with synthetic pheromone captured more weevils than traps baited with adult male weevils but the different ratios of $\mathrm{C} 1$ and $\mathrm{C} 2$ did not affect the weevils capture. In fact, traps baited with $\mathrm{C} 2$ caught more weevils than those baited with the binary blend. Also, we found that weevil's capture was not affected by dose. In general, traps baited with the synthetic pheromonal components caught more females than males.

Key words: Agave tequilana, Scyphophorus acupunctatus, pheromone bait, dosage, monitoring, mass trapping.

\section{INTRODUCCIÓN}

El picudo del agave, Scyphophorus acupunctatus Gyllenhal, es considerado un especialista en sus hábitos alimentarios, ya que solo utiliza plantas pertenecientes a las familias Agavaceae y Dracaenaceae. La mayoría de las plantas hospederas de $S$. acupunctatus tienen una importancia ecológica y ornamental, aunque esta especie también ataca algunas plantas de importancia económica, entre las cuales destacan agaváceas como el henequén y el sisal, de las cuales se obtienen fibras, y otros agaves como el pulquero, mezcalero y tequilero, de los que se obtienen bebidas alcohólicas. En México, S. acupunctatus afecta a diversas industrias de los agaves. Por ejemplo, en el cultivo del agave tequilero, Agave tequilana Weber var. azul, este insecto puede causar pérdidas de $24.5 \%$ en materia prima (Solis-Aguilar et al. 2001). En el henequén provoca pérdidas en el rendimiento de hasta un $40 \%$ (Valdés-Rodríguez et al. 2004). En el cultivo del nardo, también se considera la plaga más importante (Camino et al. 2002). Las larvas dañan directamente a la planta al barrenar la piña y pencas, mientras que los adultos, además de alimentarse de los tejidos del agave, pueden transmitir hongos y bacterias fitopatógenas (Waring \& Smith 1986, Solís Aguilar 2001), provocando una reducción de materia prima o la pérdida total de la planta (Rodríguez 1999).

El control de $S$. acupunctatus con insecticidas no ha sido exitoso debido a que las larvas y adultos se alojan dentro de los tejidos, por lo que es poca o nula la cantidad de insecticida que llega hasta el sitio en que se encuentran los picudos (Valdés-Rodríguez et al. 2004). Debido a lo anterior, es necesario buscar mejores alternativas de control que puedan ser incluidas dentro de un manejo integrado para este insecto. El manejo 
del comportamiento de los insectos plaga a través de infoquímicos es una alternativa valiosa de explorar con el picudo del agave, ya que varios estudios demuestran que estos infoquímicos pueden ser útiles en el manejo de diferentes especies de picudos (Alpízar et al. 2002, Oehlschlager et al. 2002a, b). Existen evidencias de que los compuestos volátiles de la planta hospedera atraen al picudo del agave o sinergizan la respuesta de los insectos a la feromona (Ramírez-Choza 1978, Valdés-Rodríguez et al. 2004, Altuzar et al. 2007, Ruiz-Montiel et al. 2009). Al respecto, Valdés-Rodríguez et al. (2004) identificaron químicamente cinco compuestos de extractos activos de henequén atractivos al picudo del agave. Por otro lado, Altuzar et al. (2007) identificaron seis compuestos, de los cuales en pruebas de laboratorio se determinó que el $\gamma$-terpineno fue atractivo para las hembras, el $\alpha$-pineno para machos, y el linalol y 3-careno para ambos sexos. Adicionalmente, una feromona de agregación compuesta por al menos dos alcoholes y dos cetonas, liberada por los machos del picudo del agave fue atractiva para ambos sexos (Ruiz-Montiel et al. 2003, 2008). En experimentos de campo se ha demostrado que los cuatro compuestos son atractivos a los picudos del agave, pero las mayores capturas se obtuvieron cuando se combinaron las dos cetonas (Ruiz-Montiel et al. 2008).

Aunque se tienen identificados los compuestos feromonales de $S$. acupunctatus no se han realizado estudios para determinar su efectividad para monitorear las poblaciones silvestres de picudos. Por tanto, el objetivo del presente trabajo fue evaluar el efecto de diferentes proporciones, dosis y combinaciones de las cetonas 2-metil-4heptanona (C1) y 2-metil-4-octanona (C2) en la captura del picudo del agave como un primer paso para proponer un cebo feromonal comercial que pueda usarse en un sistema de monitoreo o trampeo masivo, componente básico para un manejo integrado de este insecto.

\section{MATERIAL Y MÉTODOS}

Área de estudio. El estudio se llevó a cabo durante los meses de julio a octubre de 2007, en predios comerciales de agave tequilero, A. tequilana var. azul, en Jalisco, México. Se realizaron tres experimentos: el primero se estableció en el predio "Las Higueras" ubicado en Ameca, administrado por Promoción y Fomento del Agave, S. de R. L. de C. V. (20³4' $26.45^{\prime \prime} \mathrm{N} ; 1^{\circ} 4^{\circ} 0^{\prime}$ '41.60" O y $\left.1,235 \mathrm{msnm}\right)$, plantación de 2.3 ha y 4 años de edad, con temperatura media anual de $21.3{ }^{\circ} \mathrm{C}$, precipitación media de $864 \mathrm{~mm}$ y régimen de lluvias de junio a septiembre. Los experimentos dos y tres se establecieron en el Predio "Bajío Norte", en El Arenal, administrado por Casa Herradura ubicado entre los $20^{\circ} 42^{\prime} 44^{\prime \prime}$ a $20^{\circ} 52^{\prime} 15^{\prime \prime} \mathrm{N}$ y $103^{\circ} 37^{\prime} 4^{\prime \prime}$ a $103^{\circ} 42^{\prime}$ $45^{\prime}$ " O, con $1450 \mathrm{msnm}$, de 6 ha y 5 años de edad, con una temperatura media anual de $20{ }^{\circ} \mathrm{C}$ y precipitación pluvial media de $1103.6 \mathrm{~mm}$ (GEJ 2005).

Experimentos. Los tratamientos experimentales se evaluaron inicialmente con una trampa diseñada por Rangel Reyes (2007), la cual consiste de una cubeta de plástico 
color blanco de $5 \mathrm{~L}$ de capacidad, $21 \mathrm{~cm}$ de diámetro y $18.5 \mathrm{~cm}$ de altura, con cinco perforaciones laterales, verticales y paralelas, de $3 \mathrm{~cm}$ de ancho y $8 \mathrm{~cm}$ de altura, con una separación de la base de $3 \mathrm{~cm}$ para permitir la entrada de los picudos y $5 \mathrm{~cm}$ de la tapa. El liberador con los compuestos feromonales se colgó con un gancho de alambre por dentro del centro de la tapa de la cubeta. Se utilizaron $100 \mathrm{~g}$ de material vegetal por trampa como complemento del sistema de atracción, el cual se obtuvo de la base de pencas de agave con apariencia sana. En las trampas testigo con 10 picudos macho vivos, los $100 \mathrm{~g}$ de tejido de agave se dividieron en dos partes: $50 \mathrm{~g}$ se dispusieron dentro de la trampa y $50 \mathrm{~g}$ dentro del bote que contenía los picudos. Las paredes internas de las trampas, así como los fragmentos de agave, se impregnaron con $15 \mathrm{~mL}$ de paratión metílico al $0.3 \%$ mediante un aspersor manual.

Para el experimento 1 se usaron como liberadores tubos de centrifuga de propileno Ependorf de $0.5 \mathrm{~mL}$ de capacidad con dos orificios de $1 \mathrm{~mm}$ de diámetro en la parte superior del tubo. En el resto de los experimentos, se utilizaron liberadores tipo membrana o bolsa de $7 \times 5 \mathrm{~cm}$.

Los tratamientos se dispusieron en un diseño experimental de bloques completos al azar con cuatro repeticiones. Para los experimentos 1 y 2 la separación entre bloques (hileras dentro del cultivo) fue de $12 \mathrm{~m}$ y $10 \mathrm{~m}$ entre trampas (tratamientos), para el experimento 3 la separación entre trampas fue de $20 \mathrm{~m}$ y $21 \mathrm{~m}$ entre bloques. En todos los experimentos se utilizó un testigo consistente de 10 machos de $S$. acupunctatus, los cuales se colocaron dentro de botes de plástico transparente de $13 \mathrm{~cm}$ de alto por $11 \mathrm{~cm}$ de diámetro, con una abertura en la tapa de $16 \mathrm{~cm}^{2}$ cubierta con tela mosquitera de plástico de 15 hilos por pulgada ${ }^{2}$.

En el primer experimento se evaluaron cinco tratamientos con diferentes proporciones $(1.33: 1,2.66: 1,1: 1.5,5.33: 1$ y 1:3) de $\mathrm{C} 1$ y C2 más el testigo que incluía 10 picudos macho vivos, los compuestos fueron cargados a los tubos Ependorf en $\mu \mathrm{L}$, las cantidades de cada compuesto en los diferentes tratamientos se muestra en el Cuadro 1.

Cuadro 1. Porcentaje de hembras capturadas en trampas cebadas con diferentes proporciones de los compuestos feromonales $\mathrm{C} 1$ y $\mathrm{C} 2$, estimado por el modelo logístico con un nivel de significancia del 5\%. El experimento fue realizado en Ameca, Jalisco. Predio Las Higueras. 2007.

\begin{tabular}{lc}
\hline Tratamientos (proporciones C1:C2) & \% Hembras capturadas \\
\hline T1 1.33:1 $(60 \mu \mathrm{L}: 45 \mu \mathrm{L})$ & 87.00 \\
T2 2.66:1 $(120 \mu \mathrm{L}: 45 \mu \mathrm{L})$ & 74.50 \\
T3 1:1.5 $(60 \mu \mathrm{L}: 90 \mu \mathrm{L})$ & 78.54 \\
T4 5.33:1 $(240 \mu \mathrm{L}: 45 \mu \mathrm{L})$ & 81.25 \\
T5 1:3 $(60 \mu \mathrm{L}: 180 \mu \mathrm{L})$ & 84.35 \\
T6 testigo $(10$ Machos) & 55.87 \\
\hline
\end{tabular}


En el experimento 2 se evaluaron las proporciones 1:1, 2:1, 4:1, 1:2 y 1:4 de C1 y $\mathrm{C} 2$ a las dosis de 50,100, y $150 \mathrm{mg}$ por cebo. En el experimento 3 se evaluaron las proporciones $1: 1,1: 4$, y $0: 1$ de $\mathrm{C} 1$ y C2 a las dosis de 200, 350, y $500 \mathrm{mg}$ por cebo. Cada experimento duró 15 días. Los compuestos feromonales fueron proporcionados por FeroComps (México, D. F.). Diariamente se registró el número de picudos capturados en trampas por tratamiento. El material capturado se guardó en bolsas de plástico y los picudos macho de las trampas testigo que morían eran reemplazados por picudos vivos recolectados previamente en el mismo predio y separados por sexo en laboratorio. Para evitar el sesgo de datos, las trampas se rotaron diariamente dentro del bloque. Las trampas se colocaron a nivel del suelo en la base de la planta y dentro de la hilera del cultivo. El tejido de agave e insecticida fueron reemplazados cada 7 días. El sexo de los insectos capturados en las trampas se determinó en el laboratorio de Entomología del Colegio de Postgraduados, Montecillo, Texcoco, Edo. de México, mediante la observación morfológica del último segmento abdominal (RamírezChoza 1993).

Análisis estadístico. Los datos del número de picudos capturados por trampa fueron analizados usando el procedimiento GLM; además, cuando en el tratamiento se observó un efecto significativo se aplicaron contrastes. Mientras que para evaluar si existían diferencias en la proporción de sexos de los picudos capturados, se aplicó una regresión logística con PROC LOGISTIC; los datos fueron analizados con el paquete estadístico SAS (SAS Institute 2002).

\section{RESULTADOS}

En el experimento 1, el numero de picudos capturados fue significativamente influenciado por el tratamiento evaluado $(\mathrm{F}=3.71, \mathrm{GL}=5,15 p=0.0219)$. Las trampas cebadas con las diferentes proporciones de $\mathrm{C} 1$ y $\mathrm{C} 2$ capturaron, en promedio, un mayor número de picudos en comparación con las trampas cebadas con machos adultos (T1 $+\mathrm{T} 2+\mathrm{T} 3+\mathrm{T} 4+\mathrm{T} 5 / 5 v s$. T6) $(\mathrm{GL}=1,15, p=0.0109)($ Fig. 1$)$.

Consistentemente, en este experimento en las trampas cebadas con los compuestos sintéticos se capturaron un mayor número de hembras que de machos. Los tratamientos T1 (1.33:1), T4 (5.33:1) y T5 (1:3) capturaron la mayor proporción de hembras, mientras que las trampas cebadas con machos vivos (T6) capturaron un porcentaje similar de hembras y machos (Cuadro 1).

En el experimento 2, a las dosis de 50,100 y $150 \mathrm{mg}$ de ambas cetonas no se detectaron diferencias significativas en el número picudos capturados por las trampas cebadas con los diferentes tratamientos, incluyendo el testigo $(\mathrm{F}=0.95, \mathrm{GL}=15,45$, $p=0.5206$ ) (Fig. 2).

Los resultados obtenidos en este experimento muestran que las trampas cebadas con los compuestos sintéticos capturaron una mayor proporción de hembras. La 


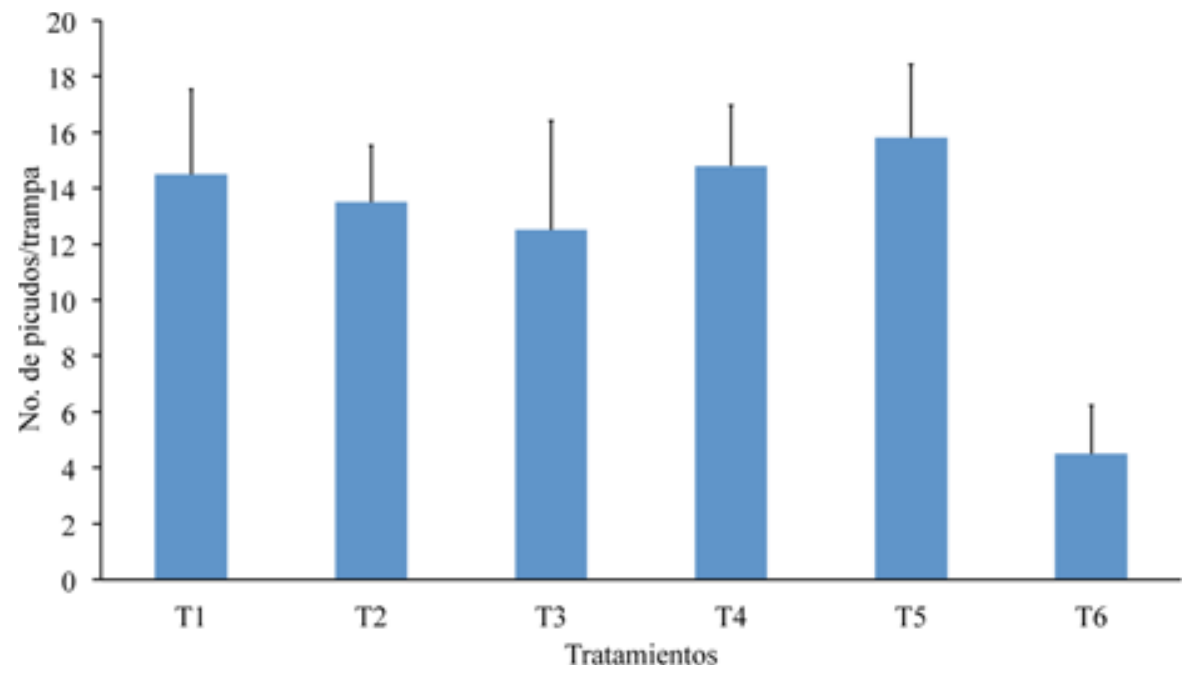

Figura 1. Promedio de picudos del agave (+ EE) capturados en trampas cebadas con los componentes feromonales $\mathrm{C} 1$ y $\mathrm{C} 2$ en diferentes proporciones. La cantidad de cada componente en los diferentes tratamientos se muestran en el Cuadro 1. Como testigo se usaron trampas cebadas con 10 picudos macho. Este experimento se realizó en el Predio Las Higueras, Ameca, Jalisco, México. 2007.

mayor proporción de hembras capturadas se logró con las trampas cebadas con las proporciones 1:2 (T4) y 1:4 (T5) con la dosis $50 \mathrm{mg}$, así como con la dosis de 150 mg en proporción de 1:2 (T14), aunque estos tratamientos no presentaron las mayores capturas conjuntas de machos y hembras, excepto el T4. Solamente los picudos adultos macho vivos capturaron igual porcentaje de machos y hembras (Cuadro 2).

En el experimento 3, el número de picudos capturados fue significativamente afectado por los tratamientos $(\mathrm{F}=10.81, \mathrm{GL}=9,27, p=0.0001)$. Al aplicar contrastes, se detectaron diferencias significativas entre los tratamientos donde se mezclaron las dos cetonas y los tratamientos que sólo contenían a la C2 a las dosis de 200, 350 y $500 \mathrm{mg}$, los cuales capturaron un mayor número de picudos (Fig. 3).

En este experimento también se capturaron más hembras que machos con los cebos feromonales sintéticos, particularmente con los tratamientos $\mathrm{T} 6(1: 4 \mathrm{de} \mathrm{C} 1+\mathrm{C} 2)$ a $500 \mathrm{mg}$, T8 y T9 de C2 a 350 y $500 \mathrm{mg}$, respectivamente. Por el contrario, el tratamiento con la menor proporción de hembras capturadas se presentó en el tratamiento que incluía picudos adultos macho vivos (Cuadro 3).

\section{DISCUSIÓN}

Las feromonas han demostrado ser una herramienta eficiente, en tiempo y forma, para monitorear y en algunos casos controlar insectos plaga, ya que generalmente sólo 


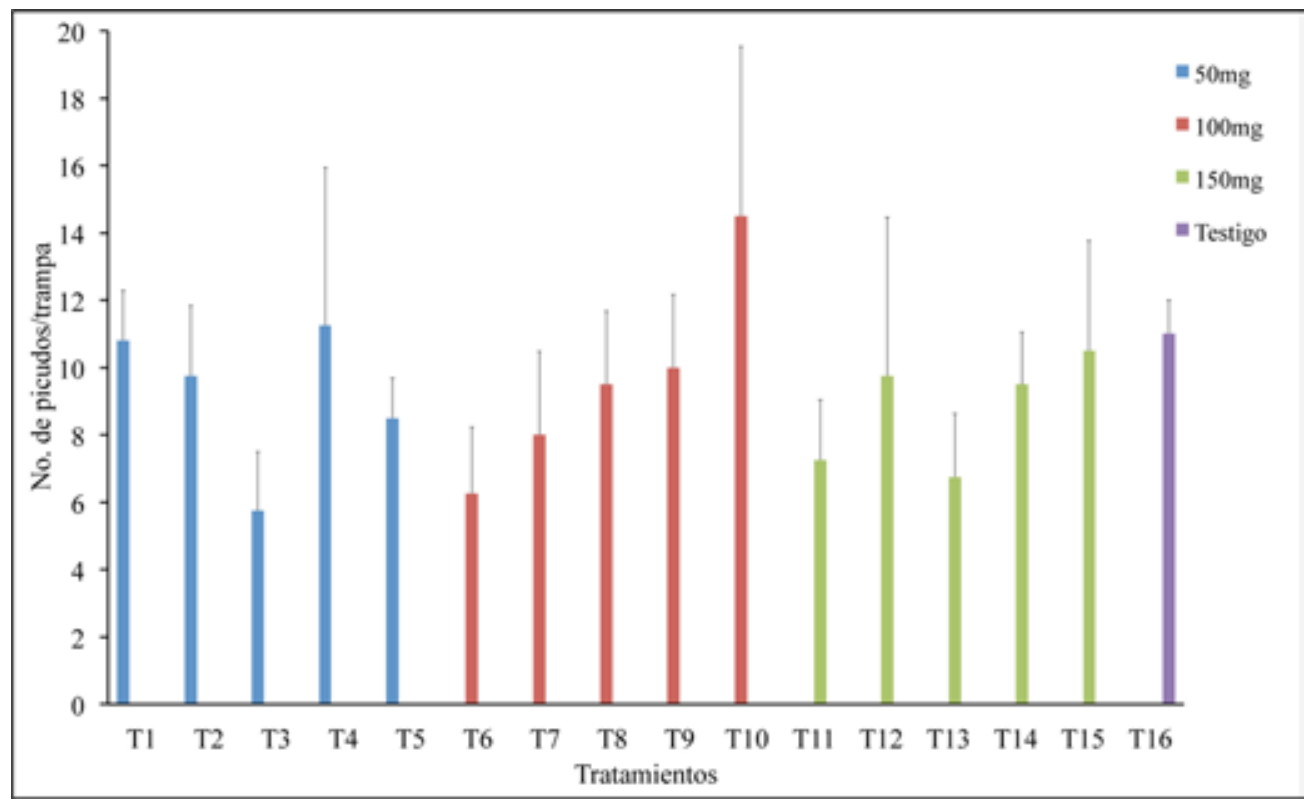

Figura 2. Promedio de picudos del agave (+EE) capturados en trampas cebadas con diferentes dosis $(50,100$ y $150 \mathrm{mg})$ y proporciones de los componentes feromonales $\mathrm{C} 1$ y C2. La cantidad de cada componente en los diferentes tratamientos se muestran en el Cuadro 2. Como testigo se usaron trampas cebadas con 10 picudos macho. Este experimento se realizó en el Predio "Bajío Norte", El Arenal, Jalisco. 2007.

atraen al insecto específico. En la familia Curculionidae comúnmente es el macho quien libera la feromona que atrae a ambos sexos (revisado por Bartelt, 1999), como ocurre en el picudo del agave, en el picudo del banano, Cosmopolites sordidus (Germar), el picudo de la caña de azúcar, Metamasius hemipterus sericeus (Olivier) y el picudo de la palma de coco, Rhynchophorus palmarum (L.) (Chinchilla \& Oehlschlager 1992, Weissling et al. 1993, Pérez et al. 1997, Ruiz-Montiel et al. 2003, 2008). En contraste, en el picudo del camote, Cylas formicarius (F.), las hembras son las que liberan la feromona, atrayendo solo a machos (Jansson et al. 1991).

En el presente trabajo se determinó que las diferentes proporciones probadas de las cetonas $\mathrm{C} 1$ y C2 no afectaron las capturas de las trampas cebadas. De hecho, las trampas cebadas con la $\mathrm{C} 2$ dieron mejores capturas que la mezcla binaria de las dos cetonas. Desde el punto de vista práctico el hecho de que un sólo compuesto sea suficiente en la atracción de los picudos resulta conveniente, ya que reduce los costos de producción de un cebo comercial. En otros casos, sin embargo, se requiere más de un componente para que la feromona sea atractiva. Por ejemplo, en el sistema de trampeo de M. hemipterus sericeus se requieren de al menos dos compuestos feromo- 
Cuadro 2. Porcentaje de hembras capturadas en trampas cebadas con diferentes proporciones y dosis de los compuestos feromonales $\mathrm{C} 1$ y $\mathrm{C} 2$, estimado por el modelo logístico con un nivel de significancia del 5\%. El experimento fue realizado en el Predio "Bajío Norte". El Arenal, Jalisco. 2007.

\begin{tabular}{lcc}
\hline Proporción de C1: C2 & Dosis $(\mathrm{mg})$ & Hembras capturadas (\%) \\
\hline T1 1:1 (25:25)* & 50 & 70.53 \\
T2 2:1 (33:17) & & 79.22 \\
T3 4:1 (40:10) & & 78.40 \\
T4 1:2 (17:33) & & 88.94 \\
T5 1:4 (10:40) & 100 & 88.55 \\
T6 1:1 (50:50) & & 64.71 \\
T7 2:1 (66:33) & & 75.26 \\
T8 4:1 (80:20) & & 68.96 \\
T9 1:2 (33:66) & 76.94 \\
T10 1:4 (20:80) & 75.87 \\
T11 1:1 (75:75) & 150 & 78.57 \\
T12 2:1 (100:50) & 71.27 \\
T13 4:1 (120:30) & 72.71 \\
T14 1:2 (50:100) & 86.77 \\
T15 1:4 (30:120) & 77.21 \\
T16 Testigo (10 machos) & 55.67 \\
\hline *antidad de cada compuesto en mg. & \\
& & \\
\hline
\end{tabular}

nales (Giblin-Davis et al. 1994, Pérez et al. 1997). En el trampeo de Ips typographus L., se requieren de dos compuestos feromonales y las mayores capturas de hembras se obtienen cuando hay una mayor proporción de uno de los componentes en el liberador (Jakus \& Blazenec, 2002). Con respecto al factor de las dosis evaluadas, los resultados del presente trabajo muestran que la dosis no afectó las capturas de trampas cebadas con la feromona de $S$. acupunctatus. En contraste, en otros escarabajos se ha encontrado una respuesta a la dosis de su feromona (Bartelt et al. 1994, Reddy et al. 2005). Por ejemplo, en Carpophilus mutilatus Erichson las trampas cebadas con 50 a $15,000 \mu \mathrm{g}$ de su feromona fueron más atractivas que el control y la captura se incrementó conforme se aumentaba la dosis (Bartelt et al. 1994).

Los resultados del presente trabajo también muestran que las trampas cebadas con los compuestos sintéticos capturan significativamente una mayor proporción de hembras que de machos en relación con las trampas cebadas con machos vivos, las cuales capturaron una proporción similar de hembras y machos. Esto sugiere que las dosis usadas afectaron el comportamiento de los picudos. Generalmente, los machos 


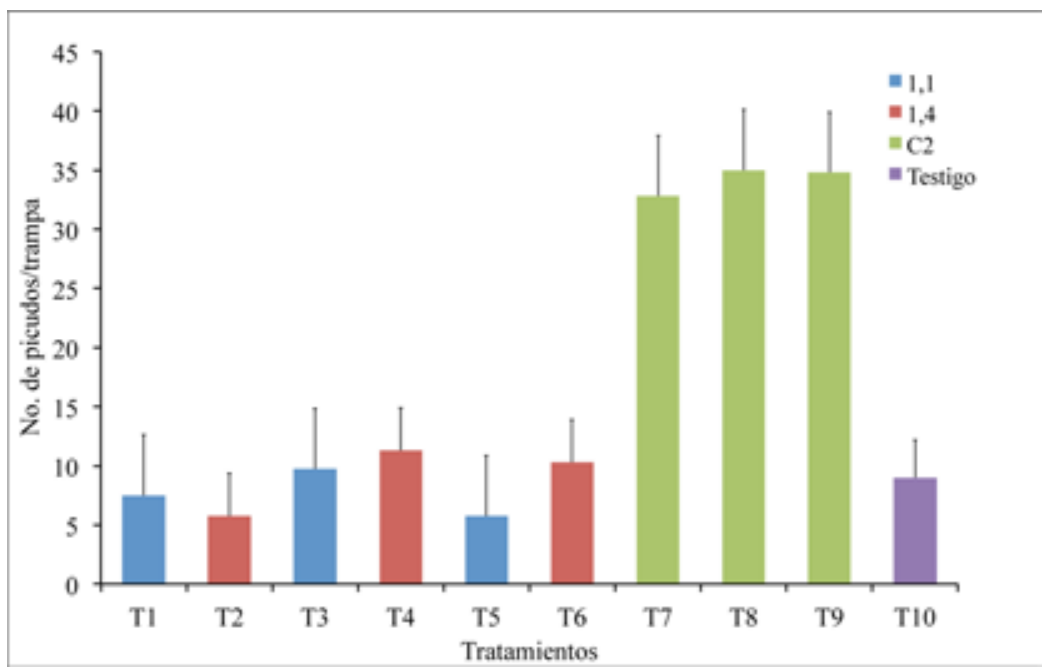

Figura 3. Promedio de picudos del agave capturados (+ EE) en trampas cebadas con diferentes dosis (200, 350 y $500 \mathrm{mg}$ ) y proporciones de los compuestos feromonales C1 y C2. La cantidad de cada componente en los diferentes tratamientos se muestra en el Cuadro 3. Como testigo se usaron trampas cebadas con 10 picudos macho. Este experimento se realizó en el Predio "Bajío Norte", El Arenal, Jalisco. 2007.

de $S$. acupunctatus liberan entre 1.4 a $11.6 \mathrm{ng} / \mathrm{hr} /$ individuo del compuesto C2 (RuizMontiel et al. 2009). El hecho de que las trampas cebadas con la feromona sintética capturen más hembras que machos ya había sido reportado en otros estudios con $S$. acupunctatus (Ruiz-Montiel et al. 2008, García Coapio 2009). Se piensa que la función primaria de la feromona de $S$. acupunctatus es la de reclutar conespecíficos de ambos sexos para colonizar la planta hospedera, sin embargo, es posible que una función secundaria de esta feromona sea de tipo sexual. Así bajo este escenario es posible que a una alta concentración de feromona los machos eviten los cebos que podrían indicar una alta densidad de machos que potencialmente serian una competencia en la búsqueda de pareja, mientras que para las hembras significaría la posibilidad de encontrar machos para aparearse. Si la feromona tiene una función sexual debe ser investigada en futuros estudios. Otra posibilidad es que a altas concentraciones de feromona los machos se fatiguen sensorialmente más rápidamente que las hembras y así dejen de responder a la feromona. La fatiga sensorial incluye dos procesos, la adaptación y la habituación, y ambos son dependientes de la concentración de feromona en palomillas (Sanders 1997). Cuando un macho de palomilla se acerca a una alta concentración de feromona se fatiga sensorialmente y deja de volar hacia la fuente feromonal (Sanders 1997). Una tercera explicación al hecho de capturar más hembras que machos sería que al momento de realizar los experimentos hubiera 
Cuadro 3. Porcentaje de hembras de $S$. acupunctatus capturadas en trampas cebadas con diferentes proporciones y dosis de los compuestos feromonales $\mathrm{C} 1$ y $\mathrm{C} 2$, estimado por el modelo logístico con un nivel de significancia del 5\%. El experimento fue realizado en el Predio Bajío Norte, El Arenal, Jalisco. 2007.

\begin{tabular}{lcc}
\hline Proporción de C1: C2 & Dosis $(\mathrm{mg})$ & Hembras capturadas (\%) \\
\hline T1 1:1 (100:100)* & 200 & 79.89 \\
T2 1:4 (40:160) & & 85.0 \\
T3 1:1 (175:175) & 350 & 78.99 \\
T4 1:4 (70:280) & & 84.24 \\
T5 1:1 (250:250) & 500 & 83.55 \\
T6 1:4 (100:400) & & 91.07 \\
T7 0:1 (0:200) & 200 & 87.06 \\
T8 0:1 (0:350) & 350 & 90.61 \\
T9 0:1 (0:500) & 500 & 92.0 \\
T10 Testigo (10 machos) & & 58.72 \\
\hline
\end{tabular}

* Cantidad de cada compuesto en mg.

habido en el campo más hembras que machos. Sin embargo, las trampas cebadas con machos vivos capturaron una proporción sexual cercana a 1:1. Adicionalmente, en plantaciones comerciales de agave tequilero de Amatitán y Ahualulco, Jal., se ha encontrado, mediante muestreo directo de plantas de agave infestadas de picudo, que la proporción sexual del picudo del agave es de alrededor de 1:1 durante todo el año (Figueroa Castro 2009).

En todos los tratamientos evaluados fue necesario colocar material vegetal en las trampas como complemento en el sistema de atracción de los picudos, ya que se ha observado que las feromonas sintéticas y los volátiles del agave, por separado, no ejercen tal poder de atracción en los picudos del agave a nivel de campo (García Coapio 2009). Al respecto, Jaffé et al. (1993) mencionan que para que la feromona de agregación del picudo del cocotero sea más efectiva es necesaria la presencia de los volátiles de la planta hospedera, ya que la liberación de feromona de agregación por curculiónidos ocurre justo cuando éstos se alimentan del sustrato (Bartelt 1999). Por su parte, Chinchilla \& Oehlschlager (1992) describen diseños de trampas para $R$. palmarum, en las cuales es esencial la presencia de la feromona de agregación y una fuente de alimento para hacer más eficientes las capturas.

Si bien en el presente estudio se ha investigado el efecto de las dosis y las proporciones de los compuestos feromonales en la captura del picudo, otros factores deben ser investigados antes de proponer un sistema operacional basado en la feromona de S. acupunctatus. Por ejemplo, se necesita conocer cuál es el efecto de la tasa de liberación del componente feromonal sobre la captura, así como la influencia del tipo y 
color de la trampa, la altura a la que se debe colocar y la densidad óptima de trampas para evitar interferencia entre ellas. También será necesario identificar los compuestos del agave que sinergizan la respuesta de los picudos a la feromona de agregación para incorporar los compuestos sintéticos al sistema de trampeo. El uso de una kairomona sintética en lugar del tejido de agave reduciría el tiempo y los costos de mano de obra asociados con el mantenimiento del trampeo. Por último, será recomendable obtener más información acerca de la biología del picudo y de la influencia de las condiciones ambientales sobre el comportamiento de $S$. acupunctatus. Hasta ahora no se sabe a qué distancia pueden desplazarse los picudos y como el clima contribuye en su dispersión. En una especie relacionada, Scyphophorus yuccae Horn., se encontró que los adultos se mueven constantemente de plantas hospederas sin floración a plantas en floración, que tienen la capacidad de desplazarse entre 10-33 m y que usan la dirección del viento para dispersarse (Huxman et al. 1997).

El hecho de haber capturado más hembras que machos y la posibilidad de usar sólo un componente de la feromona sugiere el uso potencial de dicho compuesto en un sistema de trampeo con fines de monitoreo o de trampeo masivo, dentro de un programa de manejo integrado del picudo del agave. En un sistema de trampeo masivo, el potencial para reducir la población de picudos sería mayor, si preferencialmente se capturaran más hembras que machos (Ruiz-Montiel et al. 2008).

Agradecimientos. A Tequila Sauza S. A. de C. V. y Casa Herradura, por el apoyo económico y logístico a los proyectos: "Biología, Efectividad Biológica de Insecticidas y Ecología Química del Picudo del Agave" y "Manejo Integrado de Plagas del Agave". A TAMU-CONACYT Collaborative Research Grant Program, por el apoyo al proyecto: "Developing Environmentally Friendly Management Technologies for Emerging Insects Pests of Tequila Agave". Al Colegio de Postgraduados, por el apoyo económico y facilidades otorgados en el Laboratorio del Programa de Fitosanidad en Entomología Campus Montecillo. Al Consejo Nacional de Ciencia y Tecnología (CONACYT) por el apoyo económico para realización del programa doctoral del primer autor de este trabajo. A la Compañía FeroComps por facilitar los cebos utilizados los experimentos de este estudio.

\section{LITERATURA CITADA}

Alpízar, D., M. Fallas, A. C. Oehlschlager, L. M. González, C. M. Chinchilla \& J. Bulgarelli. 2002. Pheromone mass trapping of the West Indian sugarcane weevil and the American palm weevil (Coleoptera: Curculionidae) in palmito palm. Florida Entomologist, 85: 426-430.

Altuzar, A., E. A. Malo, H. González-Hernández, \& J. C. Rojas. 2007. Electrophysiological and behavioural responses of Scyphophorus acupunctatus (Coleoptera: Curculionidae) to Agave tequilana volatiles. Journal of Applied Entomology, 13: 121-127.

Bartelt, J. R. 1999. Weevils, pp: 91-112. In: J. Hardie \& A. K. Minks (Eds.). Pheromones of non-lepidopteran insects associated with agricultural plants. CABI Publishing, Wallinford, UK.

Bartelt, J. R., R. S. Vetter, D. G. Carlson \& T. C. Baker. 1994. Influence of pheromone dose, trap height, and septum age on effectiveness of pheromones for Carpophilus mutilatus and C. hemipterus (Coleoptera: Nitidulidae) in a California date garden. Journal of Economic Entomology, 87: 667-675. 
Camino, L. M., V. R. Castrejón, V., R. Figueroa, L. Aldana \& M. E. Valdez. 2002. Scyphophorus acupunctatus (Coleoptera: Curculionidae) attacking Polianthes tuberosa (Liliales: Agavaceae) in Morelos. México. Florida Entomologist, 85: 392-393.

Chinchilla C. M \& A. C. Oehlschlager. 1992. Comparación de trampas para capturar adultos de Rhynchophorus palmarum utilizando la feromona de agregación producida por el macho. ASD Oil Palm Papers, 5: 9-14.

Figueroa Castro, P. 2009. Fluctuación poblacional y trampeo de Scyphophorus acupunctatus Gyllenhal (Coleoptera: Curculionidae) con feromona de agregación en plantaciones de agave tequilero en Jalisco. Tesis de Maestría en Ciencias. Postgrado en Protección Vegetal. Universidad Autónoma de Chapingo. Chapingo, México. 63 p.

García Coapio, G. 2009. Sistema de trampeo con feromona de agregación y volátiles vegetales para el picudo del agave, Scyphophorus acupunctatus Gyllenhal (Coleoptera: Curculionidae). Tesis de Maestría en Ciencias. Colegio de Postgraduados. Montecillo, México. 75 pp.

Giblin-Davis, R. M., J. M. Peña \& R. E. Duncan. 1994. Lethal trap for evaluation of semiochemical mediated attraction of Metamasius hemipterus sericeus (Olivier) (Coleoptera: Curculionidae). Florida Entomologist, 77: 247-255.

Gobierno del Estado de Jalisco (GEJ). 2005. Enciclopedia de los municipios de México. Jalisco. Instituto Nacional para el Federalismo y el Desarrollo Municipal. Gobierno del Estado de Jalisco (GEJ). Página de internet. http://www.e-local.gob.mx/work/ templates/ enciclo/jalisco/. Fecha de revisión 10 de enero de 2011.

Huxman, T. E., K. A. Huxman, \& M. R. Stamer. 1997. Dispersal characteristics of yucca weevil (Schyphophorus yuccae) in flowering field of Yucca whipplei. Great Basin Naturalist, 57: 38-43.

Jaffé, K., P. Sánchez, H. Cerda, N. Urdaneta, J. V. Hernández, G. Guerra, R. Martínez \& B. Miras. 1993. Chemical ecology of the palm weevil Rhynchophorus palmarum (L.) (Coleoptera: Curculionidae): attraction to host plants and to a male produced aggregation pheromone. Journal of Chemical Ecology, 19: 1703-1720.

Jansson, R. K., L. J. Mason, \& R. R. Heath. 1991. Use of sex pheromone for monitoring and managing Cylas formicarius, pp. 97-138. In: R. K Jansson \& K.V. Raman (Eds.). Sweet potato pest management: a global perspective. Westview Press, Boulder, Colorado, USA.

Jakus, R. \& M. Blazenec. 2002. Influence of proportion of (4S)-cis-verbenol in pheromone bait on Ips typographus (Col., Scolytidae) catch in pheromone trap barrier and in single traps. Journal of Applied Entomology, 126: 306-311.

Oehlschlager, A. C., C. M. Chinchilla, G. Castillo \& L. M. González. 2002a. Control of red ring disease by mass trapping of Rhynchophorus palmarum (Coleoptera: Curculionidae). Florida Entomologist, 85: 507-513.

Oehlschlager, A. C., L. M. González, M. Gómez, C. Rodríguez, \& R. Andrade. 2002b. Pheromone-based trapping of West Indian sugarcane weevil in a sugarcane plantation. Journal of Chemical Ecology, 28: 1653-1664.

Pérez, A. L., Y. Campos, C. M. Chinchilla, A. C. Oehlschlager, G. Gries, R. Gries. R. M. GiblinDavis, G. Castrillo, J. E. Peña, R. E. Duncan, L. M. González, H. D. Pierce, Jr., R. McDonald \& R. Andrade. 1997. Aggregation pheromone and host kairomones of the West Indian sugarcane weevil, Metamasius hemipterus sericeus. Journal of Chemical Ecology, 23: 869-888.

Ramírez-Choza, J. L. 1978. Reporte. Evaluación del daño, biología, comportamiento y método de control del max del henequén Scyphophorus interstialis en Yucatán. CIAPY, Yucatán, México.

Ramírez-Choza, J. L. 1993. Max del henequén Scyphophorus interstitialis bioecología y control. Serie Libro Técnico. Centro de Investigación Regional del Sureste. INIFAP-SARH. Mérida, Yucatán, México. 127 pp. 
Rangel Reyes, D. N. 2007. Compuestos volátiles del Agave tequilana Weber var. azul, que son atractivos para el picudo del agave Scyphophorus acupunctatus (Coleoptera: Curculionidae). Tesis de Maestría en Ciencias. Colegio de Postgraduados. Montecillo, Texcoco, Edo. de México. 97 pp.

Reddy, G. V. P., R. Fettkother, U. Noldt \& K. Dettner. 2005. Capture of female Hylotrupes bajulus as influenced by trap type and pheromone blend. Journal of Chemical Ecology, 31: 2169-2177.

Rodríguez G., B. 1999. La investigación en agave tequilero en el CIATEJ. In: P. Bernache y A. Ávalos C. (Eds). El agave. Unión Agrícola Regional de Mezcal Tequilero del estado de Jalisco. Guadalajara, Jalisco, México. Gaceta Informativa, 1: 2-3.

Ruiz-Montiel, C., H. González- Hernández, J. Leyva, C. Llanderal-Cázares, L. Cruz-López \& J. C. Rojas. 2003. Evidence for a male-produced aggregation pheromone in Scyphophorus acupunctatus Gyllenhal (Coleoptera: Curculionidae). Journal of Economic Entomology, 96: 1126-1131.

Ruiz-Montiel, C., G. García-Coapio, J. C. Rojas, E. A. Malo, L. Cruz-López, I. del Real, \& H. González-Hernández. 2008. Aggregation pheromone of Scyphophorus acupunctatus (Coleoptera: Curculionidae). Entomologia Experimentalis et Applicata, 127: 207-217.

Ruiz-Montiel, C., J. C. Rojas, L. Cruz-López \& H. González-Hernández. 2009. Factors affecting pheromone by Scyphophorus acupunctatus (Coleoptera: Curculionidae). Environmental Entomology, 38: 1423-1428.

Sanders, C. J. 1997. Mechanisms of mating disruption in moths, pp. 333-346. In: R. T. Cardé \& A. K. Minks (Eds). Insect pheromone research, new directions. Chapman \& Hall, New York, USA.

SAS Institute. 2002. The SAS System for windows. Version 9.0.

Solís Aguilar, J. 2001. El picudo del agave tequilero Scyphophorus acupunctatus Gyllenhal (Coleoptera: Curculionidae) en Jalisco, México. Tesis Doctoral. Colegio de Postgraduados. Montecillo, Texcoco, Edo. de México. 93 pp.

Solis-Aguilar, J. F., H. González-Hernández, J. L. Leyva, A. Equihua-Martínez, F. J. Flores- Mendoza \& A. Martínez-Garza. 2001. Scyphophorus acupunctatus Gyllenhal, plaga del agave tequilero en Jalisco, México. Agrociencia, 35: 663-670.

Valdés-Rodríguez, S., J. L. Ramírez-Choza, J. Reyes-López \& A. Blanco-Labra. 2004. Respuesta del insecto max (Scyphophorus acupunctatus Gyllenhal (Coleoptera: Curculionidae)) hacia algunos compuestos atrayentes del henequén. Acta Zoológica Mexicana (n.s.), 20: 157-166.

Waring, G. L., \& R. I. Smith. 1986. Natural history and ecology of Schyphophorus acupunctatus (Coleoptera: Curculionidae) and its associated microbes in cultivated and native agaves. Annals of the Entomological Society of America, 79: 334-340.

Weissling, T. J., R. M. Giblin-Davis \& R. H. Scheffrahn. 1993. Laboratory and field evidence for male- produced aggregation pheromone in Rhynchophorus cruentatus (F) (Coleoptera: Curculionidae). Journal of Chemical Ecology, 19: 1195-1203. 